\title{
Richard Galliano-Valdiserra
}

Université Paris-Ouest Nanterre La Défense

\section{Mowgli dans les Dolomites. Représentations paysagère et ethnographique du front italien par Rudyard Kipling en 1917}

\section{INTRODUCTION}

Incontestablement, le front italo-autrichien durant la Grande Guerre demeure, aujourd'hui encore en France, largement méconnu, malgré des échanges scientifiques de plus en plus féconds par-delà les Alpes. Cette guerre d'une sauvagerie inouie a été qualifiée de "white war" ("guerre blanche") par l'historien britannique Mark Thompson $(2008,1)$ :

Some of the most savage fighting of the Great war happened on the front where Italy attacked the Austro-Hungarian Empire. Around a million men died in battle, of wounds and disease or as prisoners. On the high Alpine sectors, the armies lived and fought in year-round whiteness ${ }^{1}$.

Ce conflit a pourtant laissé de nombreuses traces parmi les combattants, qui ont composé des témoignages écrits tels que récits, journaux de tranchée, romans.

1 "Certains des combats parmi les plus sauvages de la Grande Guerre se déroulèrent sur le front italo-autrichien. Environ un million d'hommes moururent en combattant, des suites de blessures, de maladie ou en tant que prisonniers. Dans les zones de haute montagne, les deux armées vécurent et combattirent toute une année dans la neige” (traduction de ma part). 
Parmi ces sources narratives, le reportage peu connu du célèbre écrivain Rudyard Kipling, prix Nobel de littérature en 1907, envoyé spécial du War Propaganda Bureau, chargé d'instruire et de persuader l'opinion anglo-saxonne d'une guerre juste. Pour la cause, notre écrivain a visité la ligne de front, de l'Isonzo, aux Dolomites en passant par le plateau d'Asiago, en mai 1917.

Le père de Mowgli a laissé une série originale de cinq articles ${ }^{2}$, parus dans le Daily Telegraph et la New York Tribune, entre le 6 juin et le 20 juin 1917, rassemblés sous le titre éloquent de The War in the Mountains ${ }^{3}$, témoignage qui souffre pourtant d'une faible notoriété en Italie, selon l'historien Massimo Zamorani $(2011,5)$, "un libro che ben pochi in Italia conoscono". Ces textes de propagande appartiennent bien à la littérature de guerre, à plus forte raison quand ils sont signés par un des plus grands écrivains britanniques.

Ce recueil d'articles d'un observateur, non-combattant, qui ne doutait pas du bien-fondé de la propagande, est en effet d'une extrême richesse littéraire, à la fois poétique et ethnographique, ce qui nous permet de le faire entrer pleinement dans le champ de l'histoire du sensible et des représentations de la guerre.

L'écrivain reporter, immergé dans cet étrange décor de haute montagne et de hauts plateaux, sait allier la puissance de l'écriture réaliste à la métaphore poétique, pour décrire les différents acteurs de cette guerre verticale, saisir les odeurs (le paysage olfactif) et les sons de la guerre (le paysage sonore).

Comment Kipling, par son saisissant témoignage, se mue-t-il en reporter de guerre, tout en faisant œuvre de création littéraire?

Par ailleurs, comment est décrit et représenté le paysage de guerre? Quelle est la place de l'homme dans cet univers minéral qui dicte sa loi et sa suprématie, selon une logique d'inversion anthropomorphique?

2 "The Roads of an Army" parut le 6 juin 1917; "Podgora" parut le 9 juin; "A Pass, a King, and a Mountain" parut le 13 juin; "Only a few steps higher up" parut le 16 juin et "The Trentino Front" parut le 20 juin.

${ }^{3}$ Le récit La Guerre en montagne a paru dans la Revue des deux Mondes du $1^{\mathrm{er}}$ août 1917 dans une traduction anonyme, note l'éditeur dans Kipling 2014a. L'édition anglaise originelle date de 1917; nous nous appuyons sur la réédition de 2015, pour le 150e anniversaire de la naissance de Kipling (Kipling 2015). Toutes les citations originales, dont la traduction en français vient de Kipling 2014a, seront tirées de cette réédition et déclarées dans le texte avec l'acronyme WM.

Linguce \&-2/2016

http://www.ledonline.it/linguae/ 
Enfin, dans quelle mesure cette représentation de la guerre en montagne sert-elle de prétexte à construire des stéréotypes identitaires (notamment culturels) qui contribuent à forger une italianité, en partie fantasmée?

\section{KIPLING, DU TEMOIGNAGE À LA LITTÉRATURE}

En acceptant de se rendre sur le front italo-autrichien à la fin du printemps 1917, Rudyard Kipling se mua en grand reporter qui décrivit avec une rare force d'évocation visuelle, le paysage d'une guerre de montagne.

Comment l'écrivain se fit-il reporter de guerre? L'homme que nous présentons ici est un romancier renommé, "prophéte de l'impérialisme britannique" selon l'expression de George Orwell (1942), qui se transforme en témoin oculaire d'une tragédie qui se joue sur les contours édentés des Dolomites, ces grandes cathédrales coralliennes où la Grande Guerre trouva un théâtre à sa démesure, sur les pentes décharnées du Carso, sur le plateau d'Asiago et les rives de l'Isonzo. Kipling est un écrivain artiste (et non un combattant écrivant), acteur civil de la guerre, qui manie le récit sous une forme esthétique et ne cherche en aucun cas à objectiver le sujet qui l'inspire. Si Kipling ne prit jamais part à une bataille, sa vision de la guerre est réaliste, d'un réalisme d'autant plus saisissant qu'il est tranchant et précis dans le détail, comme dans la littérature de guerre d'un Blaise Cendrars ou d'un Jean Giono. Kipling ciséle son style, comme son père sculpteur ciselait le marbre. Il note, décrit tout à la fois, mais il déborde du cadre visuel, pour créer un morceau d'architecture esthétique.

Qu'est-ce qui a pu conduire et motiver Kipling sur cette ligne de front?

Tout d'abord, Kipling œuvre pour le War Propaganda Bureau qui a été fondé dans les premières semaines de la guerre, en septembre 1914, sur ordre du chancelier de l'Echiquier, Lloyd George. Sa mission est vitale puisqu'il s'agit de mettre au service de la Grande-Bretagne tout ce que le pays compte d'artistes et de talents littéraires. Pour l'Angleterre, l'objectif est simple: présenter les Allemands comme des barbares et sensibiliser l'opinion américaine afin que les Etats-Unis entrent dans le conflit à ses côtés. Au War Propaganda Bureau à Londres, on trouve de nombreux écrivains, au total vingtcinq des plus grands noms de la littérature britannique, parmi lesquels Thomas Hardy, Arthur Conan Doyle ou H. G. Wells. Pour les historiens, la propagande moderne s'amorce bien avec la Première Guerre mondiale, mais 
de façon encore temporaire et incohérente, au départ. Au fil du conflit, la propagande se sophistique, se systématise et l'on peut dire que dans cette guerre des idées et des opinions, la Grande-Bretagne va exceller. Durant ces quatre années de guerre, le WPB publia, à travers la presse, environ 500 textes (articles, pamphlets), savamment ciblés. Mais quel rôle jouent ces écrivains? Sont-ils des propagandistes?

Dans une thése récente, soutenue en 2009, à l'Université de Londres, un chercheur, Anurag Jain, s'est penché sur les relations de Conan Doyle, Kipling et Wells avec le War Propaganda Bureau. Il en ressort que ce ne sont pas eux qui prennent les initiatives ou qui font directement de la propagande, mais bien le WPB en organisant et facilitant leurs voyages et leurs contacts sur chaque terrain d'opération, et en diffusant leurs écrits le plus largement possible. Toutefois, comme l'écrit l'essayiste François Malye dans la préface qu'il consacre à la Visite sur les trois fronts de Conan Doyle (1916; trad. fran. 2014), "il n'a pas été nécessaire de tordre le poignet de ces grands noms de la littérature" (Malye 2014, 12), car ils n'ont jamais caché au public leur position patriotique et farouchement germanophobe. En revanche, leur participation aux travaux du WPB ne sera connue que bien plus tard, en 1935.

Mieux que tout autre, Kipling incarne cette âme britannique dilatée de la gloire de l'Empire. Il est né à Bombay en 1865, au temps de la splendeur de la domination impériale et comme ses parents, il se considéra toujours comme un "anglo-indien", bien qu'il ait passé la plus grande partie de sa vie hors d'Inde.

Il faut aussi rajouter deux autres points biographiques. Kipling connait la montagne, notamment les premiers contreforts de l'Himalaya. En mars 1885, journaliste reconnu, il fut l'envoyé spécial du vice-roi, lord Dufferin, dans les zones montagneuses hostiles du Nord-Ouest de l'Inde, deux mois durant, en tant que correspondant de guerre. Kipling escalade et dévale les pentes, suit l'armée britannique d'un campement à l'autre, sous une pluie battante et dans la boue. Un chaos qu'il décrira avec précision à la fin de son Livre de la jungle (1894). Il en ressort épuisé.

Par ailleurs, comme Conan Doyle, qu'il connaît bien depuis que ce dernier lui avait rendu visite aux Etats-Unis en 1894, il a été douloureusement frappé par le destin. Les deux écrivains ont perdu leur fils à la guerre qui les fit plonger dans un désespoir inconsolable.

Le fils unique de Kipling, le lieutenant John Kipling, âgé de 18 ans, fut tué à la bataille de Loos, au nord de Lens, lors de l'offensive alliée, en septembre 1915. Le corps fut porté disparu et Kipling cherchera des années à éclair- 
cir les circonstances de sa mort. Il écrivit ces lignes pleines de culpabilité et de remords: "If any question why we died / Tell them, because our fathers lied" ${ }^{4}$ (Kipling 1919).

Il est probable qu'une des motivations qui poussérent Kipling à se mettre au service de la propagande anti-boche, fut le ressentiment contre l'ennemi qui avait emporté la vie de son enfant, mais on peut également percevoir les articles comme une dénonciation morale de la guerre. C'est bien la guerre qui a englouti son fils. Les voyages sur les lignes de front, en France à l'été 1915 et en Italie en mai 1917 et les récits qu'il livre en pâture à une opinion anglosaxonne chauffée à blanc par la propagande, correspondent sans nul doute à une démarche intérieure, cathartique et expiatoire. Cette visite des fronts, cette fausse ballade d'un soldat de la propagande, c'est le lourd fardeau d'un père à jamais inconsolable.

Enfin, il est clair que les renseignements militaires fournis par Kipling sur des lieux de première importance stratégique, servirent au commandement britannique, lorsque ce dernier envoya un corps expéditionnaire de 40000 vétérans du front occidental, en novembre 1917 en Vénétie, afin de redresser le moral et la ligne de front italienne, après la déroute de Caporetto. En moins de deux mois, cinq divisions britanniques prirent position dans les zones d'importance stratégique cruciale, le Piave à Montello, et sur l'Altopiano dei Sette Comuni pour la bataille d'Asiago, au printemps 1918.

La question de la vérité historique ne se pose donc pas dans ce type de récit, car pour dire une vérité, il ne suffirait pas de dire une vérité objective. Kipling sait bien que la propriété principale de toute guerre (a fortiori celle-ci qui n'en finit pas) consiste précisément dans le fait qu'elle modifie et distord la perception du réel. Evoquant les pentes du massif du Carso, dévastées par les obus, ce "wicked Carso" (WM, 11; "Carso maudit") aux limites des deux royaumes, Kipling décrit les innombrables fosses "some exquisitely designed by the Devil for machine-gun positions" (WM, 12) et les ruines de murs intérieurs de maisons d'un village détruit: "The place shivered with ghosts in the hot daylight as the stones shivered in the heat" (WM, 13).

Kipling témoigne avec sa subjectivité et l'assimilation poétiques de matériaux inertes (des murs) à des évanescences inquiétantes (des figures patibulaires) est sans doute pour l'auteur l'une des meilleures façons de comprendre réellement la sauvagerie de la guerre et de s'en protéger. C'est ce que Freud

4 "Si quelqu'un veut savoir pourquoi nous sommes morts, dites-leur: parce que nos pères ont menti. “

Linguce \&-2/2016

http://www.ledonline.it/linguae/ 
entendait par "vérité subjective".

A la guerre, les mots nous manquent, tous les écrivains combattants de la Première Guerre mondiale ont bien illustré ce problème de la verbalisation de l'indicible. C'est pourquoi, les faits sont reconstitués à partir d'une sémantique poétique, support d'une invention, mais garante de la vérité. Kipling se transforme en écrivain prophétique, dont la parole se veut garante de la transmission d'une mémoire vraie.

Le critique littéraire Lucien d'Azay a pu écrire, dans la préface de l'édition française, que "le pouvoir de fascination du style de Kipling tient à sa sobriété à la fois réaliste et solennelle, au mystère que dissimule sa clarté ou, plus exactement, à une évidence déguisée en mystère" (d'Azay 2014, 27). Il faut sans doute entendre le mot mystère comme l'expression d'une voix intérieure, son daimôn, qui fait de lui le ménestrel de la gloire des armes et des héros anonymes ainsi que le messager de la souffrance universelle des hommes, endossant par là même la responsabilité de témoigner.

La rhétorique chauvine, naturellement associée au récit de propagande, qui ne suggère que fausseté, comme dans la prose cocardière d'un Maurice Barrès où le verbe sonne faux, n'est certes pas totalement absente dans le récit de Kipling, notamment dans l'ultime article du recueil sur lequel nous reviendrons. Cependant, entre écriture impressionniste, quasi cinématographique et réalisme concret, le style de Kipling réussit à faire passer un souffle puissant de lucidité et de sincérité qui détourne l'écrivain de toute forme d'imposture intellectuelle. Sa puissance d'évocation et d'abstraction l'entraîne vers les contrées de la beauté minérale, à l'image du ténébreux massif des Dolomites, source d'angoisse métaphysique et d'inspiration poétique:

Love has gone out of this huge basin of the Dolomites now [...] Sometimes the discharge sounded like a triumphant whoop across the snows! Then like the fall of trees far off in the thick woods! But it was most awful when it died down to a dumbed beat no louder than the pulse of blood in one's ears after a climb, or that hint which a mountain-slide might give before it chose to move into action on its own $(W M, 30)$.

C'est ainsi que Kipling nous plante le décor de la guerre. 


\section{LE SPECTACLE DE LA GUERRE}

Les zones du front italo-autrichien visitées par Kipling, ressemblent à un théâtre antique, avec trois scènes, les Dolomites, le Carso, le plateau d'Asiago, où des héros pathétiques jouent une pièce épique dans un environnement hostile qui acquiert très vite un caractère primitif (fortes intempéries, terrain instable et isolement des combattants).

Dès les premières lignes de son article initial ("The Roads of an Army"), Kipling décrit minutieusement l'itinéraire de sa visite, qui se déroule au mois de mai, dans des conditions météorologiques supportables. Il arrive à Udine, "dans la grande plaine vénitienne", près du quartier général de l'armée, où un officier, sans doute un colonel, lui présente "avec une clarté parfaite", selon Kipling, les trois fronts où se bat l'armée italienne: l'Isonzo à l'Est qui ouvre la route de Trieste, au nord le Trentin et enfin le plateau d'Asiago, comme point de fixation. Soit près de 650 kilomètres de frontière, essentiellement constitués de montagnes, la chaîne des Alpes, où Autrichiens et Italiens ont édifié en temps de paix, sur les positions stratégiques dominant les vallées, des forteresses en ciment armé, munies de puissants canons: "Some evil-looking ridges a long way off where there was a sound of guns debating ponderously" (WM, 7), écrit Kipling dès le début du récit.

Le contexte au printemps 1917 est encore favorable à l'armée italienne qui a progressé au-delà de Gorizia à l'Est et occupe le plateau d'Asiago. Kipling comprend très vite que cette guerre est différente de celle pratiquée sur le front occidental. Il s'agit d'une guerre verticale où les hommes ne cessent d'escalader et de pratiquer l'alpinisme. Repérant la ligne de front, des Alpes juliennes à l'Est au massif des Dolomites à l'Ouest, notre reporter écrit ces lignes:

A stranger's eyes could make out nothing except one sheer rampart of brooding mountains-like giants at a hunting'all along the northern horizon. Men must be borne or broke to the mountains to accept them easily. (WM, 8)

Puis, la visite se poursuit le long de l'Isonzo, large et ondulante rivière qu'il compare à une rivière d'Inde, sans doute le Jhelum, dans la vallée du Cachemire, région qu'il a parcourue jadis. "A snow-fed river is as untrustworthy as a drunkard” (WM, 11), écrit-il encore. La comparaison n'est pas fortuite. Kipling replonge en Orient, retrouve le temps d'une fugace immersion dans sa mémoire indienne, les odeurs, les images et les sonorités d'autrefois: "The 
flavour of mules, burning fuels, and a procession of high-wheeled Sicilian carts, their panels painted with Biblical stories, added to the Eastern illusion" (ibid.).

Comme en écho à la campagne militaire d'Alexandre le Grand dans la vallée du Cachemire, en 326 avant notre ère, l'Isonzo, espace frontière de deux civilisations, acquiert une dimension mythique pour Kipling. La bataille du fleuve incarne le moment où le fléau de la balance, après avoir longtemps hésité, semble s'incliner irrévocablement vers l'Italie, juste avant la déroute de Caporetto.

Retrouvons Kipling dans le massif du Carso. Il poursuit sa visite sur les contours du mont Sabotino, qui culmine à 609 mètres, au nord de Gorizia, "blood-drenched snout that was carried, lost and recarried" (WM, 23); le voilà au pied du Monte Nero (ou Krn en slovène) qui culmine à 2245 mètres, plus au Nord, aujourd'hui en Slovénie. Il faut savoir que les lignes autrichiennes se trouvaient à l'est de l'Isonzo, qu'elles suivaient parallèlement sur les pentes du Monte Santo et du Mont Kuk. Les Italiens allaient les franchir lors de la $10^{\mathrm{e}}$ bataille de l'Isonzo qui débuta quelques jours après la visite de Kipling et dura jusqu'au 5 juin.

Le second article de la série s'intitule "Podgora", sans doute en hommage à l'écrivain de Trieste Scipio Slataper qui mourut sur le mont Podgora, appelé aussi Monte Calvario, au cours de la quatrième bataille de l'Isonzo en décembre 1915 et dont Kipling avait sans doute lu le livre que l'écrivain italien avait consacré à sa région natale, Il mio Carso, en 1912.

Puis, c'est le Trentin et les Dolomites et l'arrivée à la station de Cortina d'Ampezzo, la station de sports d'hiver à 1220 mètres d'altitude, qui avant la guerre appartenait aux Autrichiens. Kipling indique la présence de nombreux hôtels de luxe, mais dont le style 'art nouveau' ne l'enchantait guère: "This was an ex-health and pleasure resort, which of late belonged to the Austrians, who filled it with 'new art' hotels, each more villainous in design than its neighbour" (WM, 30).

Enfin, le Sud de la ligne de front et l'ascension de la vallée du Brenta jusqu'au plateau d'Asiago où, quelques mois plus tard, des troupes britanniques participèrent aux combats. Kipling décrit un paysage de collines enveloppées dans le brouillard: "Mist wrapped the plateau we were climbing. The mountains had changed into rounded, almost barrel-shaped heights, steep above dry valleys" (WM, 42).

Pour résumer, Kipling décrit une guerre d'épopée qui se déroule dans des conditions climatiques particulièrement difficiles (le froid intense en 
altitude, le brouillard, la neige et la glace, le vent, la boue et les avalanches, le soleil de plomb l'été et le manque d'eau potable). Elle a pour théâtre un environnement de montagnes escarpées, de collines et de vallées, de forêts denses et de plaines isolées, de routes qui montent en lacets, tracées à coups de dynamite dans les plis de la roche, de sentiers de mules, à flanc de falaise dont les caractéristiques compliquent singulièrement les opérations militaires.

Dans une misére épouvantable, les combattants des deux camps s'affrontent encore et encore et les malheureux civils, comme à Gorizia ou à Asiago, coincés des deux côtés d'une ligne de front perpétuellement mouvante, se retrouvent pris en étau. Pour les hommes comme pour les bêtes, la nature devient alors l'ennemi principal dont il convient de se protéger, avant même de songer à affronter l'ennemi.

Kipling ressent à fleur de peau, ces lieux épiques où s'expriment à leur paroxysme, l'amitié et la solidarité, l'effroi et la terreur, l'exemplarité et l'honneur, l'héroïsme et l'esprit de sacrifice. Des émotions et des valeurs qui font de la guerre italo-autrichienne une histoire d'hommes et de bêtes, autant que de guerriers. Notre écrivain use et abuse des métaphores, il invente et exagère, l'homme est à son aise et y prend manifestement du plaisir. Se sent-il soldat à son tour? Au milieu des combattants, il aime sentir le souffle et le bruit de la guerre.

La seule façon de témoigner, c'est d'exprimer la sensation. Seule la métaphore est à même de révéler véritablement le vécu des hommes et des animaux. Il en est ainsi quand Kipling compare le massif calcaire du Carso à un "belly of stones" ("ventre de pierres") ou bien à une "giant smithy where the links of the new Italy are being welded under smoke and flame and heat" (WM, 12; "forge géante faite de fumée, de flammes et de chaleur"). Les fils de fer rouillés des tranchées ressemblent à des "herses faites pour dévider les corps des hommes comme de la soie" ("like harrows fit to reel men's bodies out like silk", ibid.). De même, les pierres du "Carso maudit" qui sous le coup du dynamitage, volent en éclat "avec le bruit d'une compagnie de perdrix" ("stones whirred like partridges", WM, 14). On pourrait multiplier les exemples à l'excès en un nombre aussi réduit de pages. Il y a bien reconstruction des faits à partir de l'observation subjective et un peu d'exagération ne nuit pas à l'établissement de la vérité.

La métaphore apporte alors une forme de connaissance unique et intime, seule connaissance possible de la guerre, que l'on retrouve avec intensité chez Blaise Cendrars ou Jean Giono.

La comparaison avec l'écrivain provençal n'est pas fortuite et mérite que 
l'on s'y attarde. Son roman de guerre, Le Grand Troupeau, comporte des découpages par scène, avec une alternance par chapitre d'épisodes du front et d'épisodes de l'arrière. Cette succession d'images donne un aspect cinématographique à l'écriture de Giono. On ressent la même sensation à la lecture de la série d'articles de Kipling, avec cette succession de clichés, de photographies, parfois de cartes postales, comme lorsqu'il décrit la station de Cortina ou la plaine d'Udine. Kipling mène et entraîne son lecteur dans sa ballade comme lui-même, observateur de l'Empire britannique est mené par l'officier italien. Les unités spatiales sont brisées et il n'y a pas de cause et d'effet immédiat: Kipling saute d'un mont à un autre, d'une vallée à une autre sans unité temporelle. Mais, à chaque arrêt, à chaque étape, le repère temporel est réinitialisé. Ainsi, quittant les rives de l'Isonzo, son guide l'entraîne vers les hauteurs: "We have finished with stones for a little', said the officer. 'We are going to a mountain of mud. It is dry now, but this winter it never stayed quiet'” (WM, 16).

Quelle est la place du narrateur dans le récit? Kipling est présent sur chaque lieu, tant sur le front qu'à l'arrière. Il n'est pas pour autant un narrateur omniscient et omnipotent, ce n'est qu'un témoin privilégié qui se situe à l'intérieur du récit sans jamais se définir. Il n'est qu'un suiveur, certes non pas inactif, qui s'efface derrière sa caméra. Et le lecteur est comme un spectateur dans une salle de cinéma. D'où cette impression d'irréalité, dans certaines descriptions paysagères. Comment être réaliste si la guerre est irréelle? Kipling écrit: "Earth, however battered, one can make some sort of shift to walk on, but there was no more foothold than in a nightmare" (WM, 13).

La guerre est une pièce de théâtre antique et les montagnes en sont le décor. Kipling est fasciné par les montagnes et les sommets qui les singularisent, ces "seigneurs de la guerre", comme il les appelle, les monts calcaires désolés du Carso ou les cathédrales lugubres des Dolomites. Dans le récit, la montagne est bien l'acteur omnipotent de cette tragédie, comme un stratège qui fait la guerre et décide de son sort. Cette image de la montagne mouvante n'est pas rare dans l'œuvre de Kipling, puisque on la retrouve dans une célèbre nouvelle, écrite en 1888, “The Man Who Would Be King” (L’Homme qui voulut être roi), dont le cadre naturel est un paysage de haute montagne dans un pays légendaire, le Kafiristan.

Les hommes et les bêtes sortent du 'giron'de la montagne, enfantés dans ses entrailles les plus profondes. La montagne est semblable à un monstre de la mythologie qui engloutit les combattants et les recrache, pour les faire revivre à nouveau. Le combat est bien trop inégal, quand les "hommes dispa- 
raissent absorbés dans l'immensité des choses" ("they are swallowed up in the vastness of things", WM, 31).

Les métaphores sur la montagne renvoient essentiellement à un triple registre, anthropomorphique, zoologique ou fantastique.

Les mots et expressions anthropomorphiques où la montagne est renvoyée à une humanité souffrante, témoignent d'une puissance allégorique extrêmement efficace: "an agony of tortured crags", WM, 8), le Carso assimilé à un "ventre de pierres", le sol qui se met à "hoqueter" "the ground hiccupped", WM, 14), la montagne qui fait un bruit souterrain "comme un claquement de dents" ("as teeth chatter", ibid.).

Le zoomorphisme, par la présence d'un riche bestiaire, rappelle la veine poétique du conte animalier propre à l'univers de Kipling: "some dry, ragged points, like a cow's hips" (WM, 13), "the poisonous edged stones of the Carso" (WM, 14); les pièces d'artillerie qui se nichent dans l'épaisse végétation de la forêt "like elephants at noon" (WM, 23) ou encore les canons de la défense aérienne "fill the gorges with multiplied coughings more resembling a lion's roar than thunder" (WM, 17).

Enfin, il faut remarquer la présence du fantastique et l'inspiration mythologique. Les métaphores de Kipling ne dessinent pas, comme d'autres écrivains ont pu le faire, un paysage lunaire, mais un paysage qui apparaît comme une fabrique des dieux et engendré des enfers. Kipling immerge le lecteur dans un monde des origines, celui des anciennes divinités chthoniennes, comme surgies du chaos initial. Le Carso maudit c'est Chaos, la divinité primordiale, qui n'est qu'un abîme désordonné, une figure de cauchemar.

C'est tout le cosmos qui est en jeu dans le paysage de montagne décrit par l'écrivain britannique. Un spectacle d'étrangeté qui engendre une état d'angoisse et d'effroi: "The place shivered with ghosts in th hot daylight as the stones shivered in the heat" (WM, 13), "the heathen mountains in front had yet to be baptized and entered on the roll of honour" (WM, 18). La montagne a son génie propre et comme un démon qui la hante.

L'image de la forge géante ("a giant smithy") tapie dans le sein de la montagne renvoie inévitablement à Vulcain, le dieu du feu, hideux armurier qui fabrique les armes des immortels.

La guerre est un spectacle bruyant. Kipling est sensible à toutes les sonorités du paysage en guerre. Les bruits des canons de la défense aérienne qui ressemblent au rugissement d'un lion, l'ébranlement des lourds chariots siciliens sur les routes qui mènent aux sommets, les cris perçants des mules, la toux des moteurs. C'est aussi le silence d'avant l'assaut, ce recueillement qui 
présage une grande attaque. Dans l'imaginaire imprégné d'Orient de Kipling, à l'instar de l'univers baudelairien, les couleurs, les sons et les parfums se répondent. Un paysage sonore et olfactif émerge du récit.

Ce sont les odeurs et les cris des mules, autres figures centrales de cette tragédie, qui éveillent les sens de notre narrateur. Ces mules qui apparaissent sur nombre de photographies prises sur ce front, admirables et incontournables auxiliaires des hommes dans l'ascension des sommets et qui paient à l'égal des soldats un tribut très lourd. Forces de la nature, leur mort n'est pas dissociée de celle des humains, lorsque les obus font retentir un "bruit sinistre de bois, d'hommes et de mules fracassés" ("that ripe crash which tells that timber men and mules have caught it full this time", WM, 18).

C'est aussi l'odeur "de planches fraîches, de pétrole et de neige" des remontées mécaniques, qui hissent depuis le fond des vallées jusqu'à des platesformes taillées dans le roc, des corbeilles en treillis d'acier.

Enfin, la guerre peut être, paradoxalement, une fête. Emerge du récit de Kipling un paysage sonore autre que le son perçant d'une trompe d'automobile, l'écho sourd des canons qui résonne dans les vallées, ou le cri des bêtes apeurées. Kipling découvre dans les Dolomites les concerts d'orchestre des régiments italiens qui rivalisent avec ceux d'en face, l'Autriche toute proche, pour offrir des mélodies qui résonnent dans toute la vallée: "They brayed, they neighed, and they roared; the bandsmen's faces puckered with mirth behind the brasses" (WM, 38-39). Même la montagne s'encanaille: "And the mountains faithfully trumpeted forth their insults all over again” (WM, 39).

Dans cette langue imaginaire, le récit de propagande vient se mêler. Ce reportage sur le terrain, à destination d'un public anglo-saxon sensible à l'épopée guerrière, célèbre l'identité italienne, une identité autant réelle que fantasmée, pour des raisons que nous allons exposer.

\section{LA GUERRE, FABRIQUE DE L'ITALIANITÉ}

L'heure est à la propagande et Kipling s'y engage résolument. N'a-t-il pas pataugé dans la boue des tranchées françaises, lorsqu'en juin 1915, il s'enrôla déjà comme chroniqueur de guerre, afin de persuader l'opinion américaine du bien-fondé d'un engagement militaire? Sur ce front occidental, l'écrivain, âgé de 50 ans, auréolé de gloire littéraire, avait partagé le quotidien des soldats alliés et vit la cathédrale de Reims en ruine. Il fut bouleversé, par l'état de 
désolation d'un tel édifice, "comme si la chute des pierres annonçait la barbarie humaine, commise et à venir", note ainsi l'écrivain Olivier Weber dans la préface qu'il consacre au recueil d'articles de 1915, La France en guerre (2014, 13).

Les analogies entre les deux séries d'articles, écrites à deux ans d'intervalle, sont fortes: exaltation du patriotisme, haine de l'ennemi (le "Hun", le "Boche", le "Barbare"), glorification de l'art et de la conduite de la guerre, amour des hommes et des bêtes, fascination des paysages en bataille. Kipling sait ce que l'on attend de lui, et ce travail de reporter, il le fait sans fausseté.

En Italie, en 1917, Kipling a bien conscience que le Bureau de Propagande attend de lui qu'il redonne le moral à une opinion publique britannique, lasse de la guerre, dans cette année incertaine où, comme l'a écrit l'historien Jean-Jacques Becker (1997, 87), les hommes et les sociétés étaient "fatigués de la guerre".

Pour autant, tout romancier qu'il soit, Kipling dédiant son récit à la gloire des hommes, est fondamentalement un témoin, qui se mue au fil de la plume, en passeur d'un temps suspendu, au nom d'une mémoire collective. Il en est ainsi lorsqu'il décrit la campagne italienne:

The landscape, picked out in all the greens of spring, was that of early Italian holy pictures - the same isolated, scarred hummocks rising from enamelled meadows or drifts of bloom into the same elaborate entablatures of rock, crowned by a campanile or tufted with dark trees. (WM, 16)

L'écrivain réveille ici la mémoire d'une nation, par le biais d'un témoignage impressionniste qui se déploie dans un imaginaire collectif, comme s'il voulait souligner la pérennité d'un peuple, à l'ascendance prestigieuse.

En donnant une image méliorative de ce peuple combattant, défendant avec âpreté une terre harmonieuse et colorée, sacralisée par la peinture des artistes du Moyen-Age et de la Renaissance, Kipling se fait également passeur d'espace, au nom d'intérêts diplomatiques bien compris. Il s'agit autant d'impressionner que de convaincre et d'émouvoir.

La référence à la peinture italienne dans l'évocation d'un paysage agraire empreint de sainteté n'est pas hasardeuse. Fils d'artiste, Kipling connaît l'art occidental. A-t-il à l'esprit la peinture libératrice d'espace d'un Giotto, les coloris variés et délicats d'un Simone Martini ou bien encore la lumière douce et généreuse de l'admirable campagne siennoise, peinte par Ambrogio Lorenzetti? 
Dans son aspect de fragment d'un monde jusque-là préservé, la campagne italienne n'en a que plus de prix. Il faut la défendre et la sanctuariser. Il sait parfaitement que les vibrantes insistances à la gloire de la Rome antique et au génie créatif d'une nation d'artistes, vont directement toucher la sensibilité de ses compatriotes.

N'oublions pas que les Anglais ont toujours eu, depuis le XVIe siècle, une curiosité naturelle pour ce pays méditerranéen qui incarne par ses richesses paysagères et artistiques une éternelle beauté. Dans 'le grand tour', les élites européennes choisissaient l'Italie depuis la Renaissance, parce qu'elle était le point de passage obligé de tout humaniste qui se respectait. Mais à l'instar des autres peuples du nord de l'Europe, les élites britanniques ont aussi construit leur propre vision stéréotypée sur ces populations latines, "the excitable Latin" (WM, 45), réputée versatile, démonstratif et impatient. Le récit mêle ainsi le cliché le plus convenu à la vérité ethnographique.

A la manière de rattacher l'histoire tragique d'une nation en guerre à son prodigieux passé, le récit de propagande se transforme en un roman national aux accents lyriques. Kipling redevient pour un moment, un barde des temps anciens, chantre de la race italienne, qui doit rassurer ces compatriotes britanniques des vertus morales et guerrières des héritiers de la Rome impériale: "When one looks at the faces of their generals, chiselled out by war to the very cameos of their ancestors under the Roman eagles, one inclines to the second" (WM, 46).

Le général Cadorna qu'il cite devient un Imperator des temps modernes, tel Marius ou César combattant les tribus germaniques, aux confins du monde civilisé.

Sur les contreforts de Alpes, comme autrefois sur les pentes de l'Himalaya, Kipling imagine bien une lutte titanesque entre deux forces, dont l'une représenterait la civilisation et l'autre la barbarie. Incidemment, c'est le début d'Armageddon, l'ultime bataille décrite dans la Bible. Si le cœur de l'Italie s'arrête de battre, si son armée est vaincue, c'est toute une civilisation brillante, mère de l'Europe chrétienne, qui est menacée de disparaitre: "They are fighting now, as all civilisation fights, against the essential devildom of the Boche" (ibid.).

Dans cette guerre, c'est bien à la jeunesse que Kipling voue un hymne. De jeunes soldats qui sont la fine fleur de ce que l'Italie peut offrir de mieux en sacrifice, qu'il compare à des légionnaires romains sur une frise triomphale: "They have their own pattern of steel helmet, which differs a little from ours, and gives them at a distance a look of Roman Legionaires on a frieze of 
triumph" (WM, 10). Cette jeunesse, ce sont les Alpini qui l'incarnent, auxquels l'auteur rend hommage, lorsqu'il évoque le massif du Tofane, dans les Dolomites. Ces régiments d'alpins qui forment les troupes d'élite de l'infanterie italienne sont spécialisés dans l'architecture militaire et Kipling loue leur culture de la montagne, leur endurance, leur force de caractère collective. Il est probable qu'à travers les faces "joyeuses d'une troupe de jeunes démons" ("steady-eyed young devils", WM, 34), il ait vu le visage de son fils, jeune officier d'une troupe d'élite, les Irish Guards, et disparu au combat.

Si Kipling décrit l'Italie comme une nation d'artistes, il la présente surtout comme un peuple d'ingénieux et "inventifs" artisans. Pour faire la guerre, le soldat italien doit avant tout se transformer en maçon et en terrassier. La réputation de l'Italien maçon et tailleur de pierre n'est plus à faire. Cette image participe d'ailleurs de la construction identitaire d'une nation bâtisseuse, comme au temps où les légions romaines conquérantes construisaient autant de routes, de ponts, d'aqueducs en Europe et ailleurs. Et le lecteur retrouve dans cet hommage collectif à une nation d'habiles artisans, la dette du maçon spéculatif (Kipling était franc-maçon) à la maçonnerie opérative.

Tout au long du récit, notre narrateur traduit son étonnement et son admiration pour les travaux de maçonnerie, de construction d'abris, de forts, de conduites d'eau forcées, de radiers, de routes, de fils d'acier tendus à travers les précipices, de chemins de fer à crémaillère, de creusement de tranchées dans une roche impossible à casser, sauf à coup de dynamites, sur ces terribles pentes qui tuent les reins d'un homme en si peu de temps.

The Italian trenches are cut through snow that melts, into packed snow that never melts, into packed snow that never softens; and where the snow cannot lie on the sheer rocks, they are blasted in and out among the frost-ridden rubbish of the mountain crest. (WM, 27)

D'authentiques travaux d'Hercule, dans un environnement qui impose aux survivants, sa loi d'airain.

En définitive, je me suis efforcé de vous présenter un récit assez singulier, où ce grand voyageur, qui a traversé toutes les mers, croisé tant de cultures et de peuples, devient reporter de guerre en Italie du nord, au service de la Couronne. Le grand Kipling nous livre un morceau d'architecture qui sert la propagande d'Etat, mais entre également dans le registre de la littérature de guerre. De l'évocation de cette guerre verticale, il en ressort une tragique et douloureuse beauté. Kipling a déjà la voix brisée lorsqu'il écrit ses lignes, en 
mai 1917, et malgré le ton souvent lyrique de sa prose, il ne connaît plus la tranquillité d'esprit. Si la mort accompagne le soldat à chaque pas, les morts nous apparaissent dans le texte, sous une forme euphémique, par l'évocation spectrale des grandes hécatombes, des corps fracassés et ensevelis sous la montagne, qui leur sert de sépulcre.

On est en droit de se demander si cette ballade parfois pittoresque sur la ligne de front italo-autrichienne, n'est pas au fond l'errance d'un homme au pays des morts. Alors qu'à travers cette épreuve collective, une nouvelle Italie est en train de renaitre, Kipling, lui, n'a jamais pu se relever. S'il arrive encore à convaincre, il n'a plus de certitudes. A l'issue de la guerre, c'est bien dans les cimetières militaires qu'il livrera les affres de son âme vagabonde à un deuil infini ${ }^{5}$.

\section{BIBLIOGRAPHIE}

\section{Sources primaires}

Conan Doyle, Arthur. 1916. A Visit to Three Fronts: Glimpses of the British, Italian and French Lines, New York: George H. Doran Company, 1916.

Conan Doyle, Arthur. 2014. Visite sur les trois fronts: aperçu des lignes britanniques, italiennes et françaises, préface de François Malye, Paris: Les Belles Lettres.

Giono, Jean. 1931. Le Grand Troupeau. Paris: Gallimard.

Kipling, Rudyard. 1888. "The Man who would be King”, dans The Phantom Rickshaw and other Eerie Tales. Allahabad: Wheeler.

Kipling, Rudyard. 1915. France at War. On the Frontier of Civilization. London: MacMillan and Co.

Kipling, Rudyard. 1917. The War in the Mountains. New York: Doubleday.

Kipling, Rudyard. 1919. "Common Form", dans The Years Between. London: Methuen.

Kipling, Rudyard. 2011. La guerra nelle montagne, Impressionni dal fronte italiano, a cura di Massimo Zamorani, Milano: Ugo Mursia Editore.

${ }^{5}$ Kipling rejoignit après la guerre, l'Imperial War Graves Commission (Commission impériale des sépultures militaires), responsable des cimetières de guerre britanniques qui jalonnent la ligne du front ouest.

Linguce \&-2/2016

http://www.ledonline.it/linguae/ 
Kipling, Rudyard. 2014a. La Guerre en montagne. Dans Sur la guerre, 1914-1919. Edition préfacée et annotée par Lucien d'Azay. Paris: Pocket.

Kipling, Rudyard. 2014b. La France en guerre, préface d'Olivier Weber. Paris: Les Belles Lettres.

Kipling, Rudyard. 2015. The War in the Mountains. London: Uniform Press Ltd.

Slataper, Scipio. 1912. Il mio Carso. Firenze: Libreria della Voce.

\section{Sources secondaires}

Becker, Jean-Jacques. 1997. 1917 en Europe, L'année impossible. Paris: Editions Complexe.

d'Azay, Lucien. 2014. "Préface”. Dans Rudyard Kipling 2014a. Sur la guerre, 19141919. Paris: Pocket.

Jain, Anurag. 2009. The Relationship between Ford, Kipling, Conan Doyle, Wells and British Propaganda of the First World War. Queen Mary: University of London.

Malye, François. 2014. "Préface". Dans Arthur Conan Doyle, Visite sur les trois fronts. Paris: Les Belles Lettres.

Orwell, George. 1942. "Rudyard Kipling". Horizon, February (http://orwell.ru/ library/reviews/kipling/english/e_rkip 1 décembre 2016)

Thompson, Mark. 2008. The White War. Life and Death on the Italian Front 19151919. London: Faber and Faber.

Weber, Olivier. 2014. "Préface”. Dans Rudyard Kipling. 2014b. La France en guerre. Paris: Les Belles Lettres.

Zamorani, Massimo. 2011. "Introduzione”. In Rudyard Kipling. 2011. La guerra nelle montagne. Impressionni dal fronte italiano. Milano: Ugo Mursia Editore.

\section{ABSTRACT}

The British writer Rudyard Kipling left an extremely striking testimony of his visit to the Italian-Austrian front in May 1917. In the form of five articles of propaganda, published in English and American newspapers, the famous writer described the conditions of life and battle of Italian soldiers waging a 'vertical'war in the mountains. Indeed the writer-reporter, immersed in this strange mountainous landscape far away from his Indian homeland, combines the power of realistic writing, elements of natural history and zoological metaphors to describe the different protagonists in this war (the visual land- 
scape), its smells (olfactory landscape) and its sounds (soundscape). Kipling also took the opportunity to build a history of representations of a fictitious Italianity. 